\title{
JERARQUÍA ORACIONAL EN LA GRAMÁTICA FUNCIONAL DE LA ESCUELA DE OVIEDO
}

\author{
SENTENCE HIERARCHY IN THE FUNCTIONAL GRAMMAR OF THE OVIEDO SCHOOL \\ Juan Carlos Tordera Yllescas \\ Universitat de València
}

Resumen

Dentro de la gramática tradicional, la única distinción jerárquica de la oración que se ha realizado es aquella que separa el sujeto del predicado. En el funcionalismo, tampoco se ha avanzado mucho en cuanto al establecimiento de la jerarquía oracional. La única excepción reseñable es un trabajo del maestro Salvador Gutiérrez. A nuestro juicio, aunque el trabajo es minucioso y muy detalle, parte de un supuesto que difícilmente se puede sostener: la oración es una categoría sinonímica a la frase verbal. Nosotros defenderemos que la oración es una estructura bimembre, a saber, constituida por un sujeto y un predicado. Aceptando esta estructura, se tratará de ofrecer una jerarquía sintáctica que dé cuenta del distinto comportamiento que presentan los argumentos verbales, los elementos no exigidos, el sujeto y las funciones extraoracionales. Asimismo, trataremos de dar cuenta de estructuras coordinadas que, hasta el momento, han recibido poca atención y que parecen validar nuestra propuesta.

Palabras Clave: sintaxis funcional, jerarquía oracional, sujeto y predicado, coordinación
Abstract

According to the view of traditional grammar, the only hierarchical distinction in the sentence is the one between subject and predicate. Within the framework of European Functionalism, not a lot of progress has been made, as far as the establishment of the sentence hierarchy is concerned, the only remarkable exception being a work by Salvador Gutiérrez. Even though Gutierrez's work is, indeed, very accurate and detailed, it is based on the assumption that the sentence has the same category as the verb phrase; such an assumption is, in our view, difficult to maintain. We will defend that the sentence is a two-member structure, consisting of a subject and a predicate. Assuming this structure, we will posit a different syntactic hierarchy. Our hierarchy takes account of the different behaviours shown by verbal arguments, optional complements, subject and the extra-sentential functions. In addition, we will try to delve into a group of coordinated structures which have received little attention so far and whose behaviour seems to validate our hypothesis.

KEY WORDS: coordination, functional syntax, subject and predicate, sentence hierarchy 


\section{INTRODUCCIÓN}

Tradicionalmente, se ha considerado que la sintaxis es el estudio de la lengua que da cuenta de cómo se organizan los elementos de la lengua, lo que vale tanto como afirmar que la sintaxis se ocupa de la jerarquización de las unidades lingüísticas. Así pues, la jerarquía debería ser una relación fundamental para el estudio de la sintaxis.

No obstante, en la tradición funcionalista de la Escuela de Oviedo, al establecimiento de la jerarquía oracional no se le ha prestado una atención meritoria, salvo algunas contadas excepciones a las que nos referiremos. A nuestro entender, esta debe ser una cuestión fundamental de cualquier teoría sintáctica que podría dar cuenta, por otro lado, de algunos hechos que hasta el momento no han recibido mucha atención. El objetivo del presente trabajo es proponer una posible jerarquía de la oración y dar cuenta de algunos hechos sintácticos hasta el momento ignorados.

Para ello, en primer lugar, se realizará un breve repaso de las principales aportaciones funcionalistas para la delimitación de la jerarquía oracional. En este recorrido, se repasarán las posturas de Alarcos Llorach, Martínez, Hernández Alonso y, especialmente, de Gutiérrez Ordoñez. Consideramos que este último autor ofrece uno de los más brillantes estudios en este campo, aunque creemos que su propuesta no está exenta de problemas.

Finalmente, en el último apartado, ofreceremos nuestra propuesta de jerarquía oracional con la que esperamos solucionar los problemas planteados en las anteriores propuestas e, incluso, dar respuesta a problemas que hasta el momento han sido desatendidos.

\section{UN BREVE REPASO SOBRE LA JERARQUÍA ORACIONAL}

En los estudios ofrecidos por Alarcos Llorach (1980 [1970]) no existe una dedicación especial a delimitar la jerarquía oracional. Implícitamente, el maestro funcionalista acepta la división tradicional de la oración formada por un sujeto y un predicado, dado que, entre los complementos del predicado, excluye el sujeto (Alarcos Llorach 1980 [1970]: 148-162). Es más, al considerar que una oración es la unión de un sujeto y un predicado y al negarle el carácter oracional a las construcciones de infinitivo, se ve obligado a crear una nueva etiqueta gramatical (esto es, el adyacente temático) para aquellos elementos que otros gramáticos considerarían simplemente el sujeto de la oración de infinitivo (Alarcos Llorach 1980 [1970]: 195-196): «Al llegar la primavera, Juan marchará».

En la gramática de Alarcos Llorach (1994), se introduce una innovación teórica al diferenciar entre el sujeto gramatical y el sujeto explícito o léxico. El primero sería aquel que vendría dado por los morfemas verbales, mientras que el segundo, que es un elemento optativo, vendría representado por un sintagma explícito.

\footnotetext{
"cuando el sujeto gramatical expresado por el morfema personal incluido en la terminación del verbo no hace una referencia inequívoca en la situación de habla, se agrega un adyacente que especifica la designación de esa persona y que denominamos sujeto explícito o léxico. Su presencia es, pues, optativa, ya que para que haya oración basta la existencia del sujeto gramatical. Así, al decir Escribió, proferimos una oración, pues en esa forma verbal hay un sujeto gramatical (tercera persona del singular) del cual se predica la noción «escribir» significada por el signo léxico. Cuando interesa
} 
precisar la alusión real de esa tercera persona, se añade un sustantivo (o unidad equivalente) que funciona como sujeto explícito, diciendo El niño escribió, El maestro escribió, La secretaria escribió, etc.». (Alarcos Llorach 1994: 266).

De esta forma, Alarcos establece que toda oración está formada por un sujeto y un predicado. Las denominadas oraciones impersonales serían solo casos en los que existe un sujeto gramatical, pero que no pueden presentar ningún sujeto explícito.

Respecto a otros autores funcionalistas posteriores, la atención a la jerarquía oracional ha recibido tratamientos dispares: algunos han continuado la senda señalada por el maestro funcionalista y otros, en cambio, han abierto nuevos horizontes. Así por ejemplo, en las obras de Martínez (1994a, b y c), no se dedica ninguna atención especial a la jerarquía oracional. Grosso modo, parece asentir la jerarquía oracional propuesta por la tradición gramatical así como por Alarcos.

En la obra de Hernández Alonso (1996 [1984]), existe un gran interés en explicitar las unidades jerarquizadas de la lengua (v.gr.: diálogo >, monólogo $>$ parágrafo $>$ oración $>$ nexus > sintagma > palabra > morfema), así como en ofrecer un nuevo avance teórico en la estructura del nexus. Por diferentes motivos, Hernández Alonso (1996 [1984]: 74-80) considera que todo nexus está formado por un sujeto $\left(\mathrm{SN}_{1}\right)$ y un predicado (SV). En este punto, sigue, por tanto, la tradición gramatical al considerar el nexus como una estructura bimembre. La innovación radica en la estructura del predicado o SV, en su terminología. La jerarquía que propone para el nexus es la siguiente (Hernández Alonso 1996 [1984]: 79).

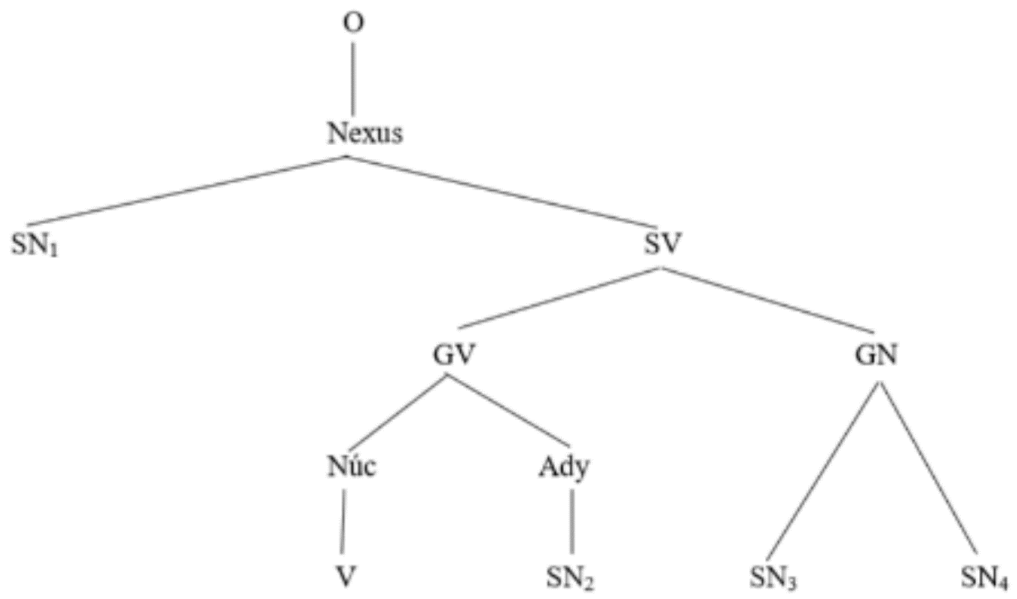

La macrofunción $\mathrm{SN}_{2}$ se caracteriza por ser «adyacente directo del verbo, responder a una función semántica común, es exigida léxico-semánticamente por los contenidos de determinados verbos [...] y, salvo en el caso del suplemento, coincidente en la conmutación» (Hernández Alonso 1996 [1984]: 83). Dicho macrofunción daría cuenta de los objetos directos, atributos y suplementos. El $\mathrm{SN}_{3}$ haría referencia al objeto indirecto, mientras que el $\mathrm{SN}_{4}$ abarcaría a los complementos circunstanciales.

A nuestro juicio, la propuesta de Hernández Alonso (1996 [1984]) ofrece la ventaja de que las distintas funciones oracionales están jerarquizadas en distintos niveles, con lo que se cumple con el requisito con el que se daba comienzo a este trabajo: no es posible que 
todas las funciones ocupen el mismo nivel jerárquico, si el nivel de incidencia es distinto. Por tanto, las funciones deben ser jerarquizadas y este parece ser el objetivo de este maestro funcionalista.

Sin embargo, a nuestro entender, se pueden realizar algunas objeciones a este modelo teórico, a saber:

a) El maestro funcionalista rechaza la propuesta de Alarcos por la que se diferencia un sujeto gramatical frente a un sujeto léxico. En palabras de Hernández Alonso (1996 [1984]: 90), no tiene sentido decir que «el sujeto gramatical reside en el morfema verbal cuando en muchísimos casos -todos los de tercera persona- éste va marcado por un signo cero, y su referencia es indeterminada». Al hacerlo, el autor deja sin resolver el problema de las denominadas oraciones impersonales. El autor debería aceptar que el nexus no siempre es bimembre, dado que existen "nexus" que no presentan el primer actante.

b) Es discutible la jerarquía que ocupa el $\mathrm{SN}_{3}$ en este modelo teórico. Si bien Hernández Alonso (1996 [1984]) asume que, salvo el suplemento, la macrofunción $\mathrm{SN}_{2}$ se caracteriza por poder ser conmutada por un pronombre átono, no incluye dentro de esta macrofunción al objeto indirecto (pese a que se puede conmutar por un pronombre átono). Las razones aducidas son de corte semántico ${ }^{1}$. Hernández Alonso (1996 [1984]: 84) afirma que la relación semántica del $\mathrm{SN}_{3}$ con el verbo «es menos intensa que la del $\mathrm{F}_{2}$; y sólo con muy pocos verbos es elemento exigido lexico-semánticamente [sic]». El rasgo de relación intensa es poco menos que discutible y, por otro lado, no es infrecuente que el objeto indirecto sea obligatorio y aparezca sin la necesidad de la existencia de un objeto directo (v.gr.: «Me encantan las patatas fritas»), frente a lo que apunta el autor. Además, dicha definición desdibujaría la distinción entre el objeto indirecto y el dativo.

c) Ni funcional ni categorialmente, se entiende la existencia de una función o constituyente como GN. Hernández Alonso (1996 [1984]: 80) afirma que la «relación entre estos dos sintagmas no es de adyacencia ni incidencia entre sí, sino de ambos hacia el GV». Es difícil de entender cómo es posible que un objeto indirecto y un circunstancial constituyan un constituyente unitario que incida sobre el SV. Por otra parte, al incluir en el $\mathrm{SN}_{4}$ los denominados suplementos inherentes de Alarcos (Hernández Alonso, 1996 [1984]: 84), se puede afirmar que la jerarquía de Alonso no tiene en consideración la obligatoriedad de los complementos verbales.

d) Hernández Alonso (1996 [1984]: 82) considera que los atributos oracionales o los moduladores oracionales (v.gr.: «tal vez») son complementos de oración. Son elementos marginales y se sobreentiende que afectarían a toda la oración. En contraste, al hablar de los complementos del nexus, menciona los $\mathrm{SN}_{2}, \mathrm{SN}_{3}$ y $\mathrm{SN}_{4}$. Sin embargo, estos elementos están subordinados al SV. Así pues, se podría plantear la posibilidad de que existan elementos marginales directamente dominados por el nexus.

\footnotetext{
1 Paradójicamente, López García (1996), gramático cognitivista, considera que el atributo, el objeto directo y el indirecto constituyen una macrofunción atendiendo a un criterio formal: todos ellos se pueden sustituir por pronombres átonos.
} 
Un último estudio funcionalista que es obligatorio mencionar es el meritorio trabajo de Gutiérrez Ordóñez (1997a). Sin lugar a dudas, este es el trabajo más detallado y elaborado con que cuenta no solo la gramática funcionalista, sino toda la gramática hispánica en referencia a la jerarquía oracional. Es un trabajo en el que a cada función le corresponde un estrato o nivel sintáctico, atendiendo, principalmente, al grado de obligatoriedad y el elemento sobre el que incide. Por otro lado, amparándose en las tesis tesnerianas, en el trabajo señalado, se parte del supuesto de que el sujeto es un argumento más del verbo. Por tanto, las oraciones impersonales no son una excepcionalidad dentro de su teoría. Se trataría, en su mayoría, de verbos ceroactanciales, esto es, verbos que no exigen ningún argumento. Consecuentemente, el autor no ve diferencias entre el concepto de oración y el de frase verbal (FV). Ambos serían conceptos sinonímicos.

Para Gutiérrez Ordóñez (1997a), el primer nivel oracional estaría ocupado por los argumentos; posteriormente, se situarían los aditamentos y, finalmente, los circunstantes. En el ámbito extraoracional, se situarían los atributos oracionales (o atributos/marcadores de modalidad lingüística) y, por encima de estos elementos, incidirían sobre el enunciado lingüístico los marcadores de modalidad pragmática, con lo que se obtendría el enunciado pragmático. En un nivel superior, se situaría el verbo enunciativo sobre el que incidirían los diferentes aditamentos y circunstantes de este verbo. Grosso modo, la jerarquía oracional es representada por Gutiérrez Ordóñez (1997a: 422) del siguiente modo:

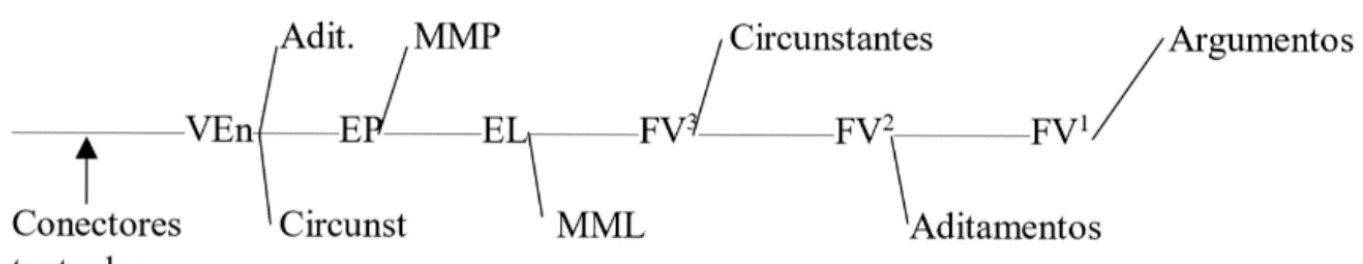

textuales

Etiquetas:

VEn: Verbo enunciativo

EP: Enunciado pragmático.

MMP: Marcador de modalidad pragmática
EL: Enunciado lingüístico

MML: Marcador de modalidad lingüística

FV: Frase verbal

Aunque algunos elementos no están exentos de polémica (v.gr.: la existencia de un verbo subyacente de carácter performativo), es innegable que el maestro Gutiérrez Ordoñez no deja elemento sintáctico alguno fuera de la jerarquía oracional. Por ello, nos parece un modelo meritorio y loable. No obstante, existe un punto cuestionable que puede suponer una fisura importante a este modelo de jerarquía oracional: es discutible identificar el concepto oración con la frase verbal o, dicho de otro modo, no nos parece un criterio suficiente (aunque sí necesario) la existencia de un verbo para que exista una oración. Gutiérrez Ordóñez (1997b: 62) recuerda los siguientes criterios tesnerianos por los que cabría desechar la estructura bimembre de la oración, esto es, razones por las que habría que asumir que el sujeto es un complemento más del verbo: 
a) El sujeto puede faltar y el predicado, no. Por tanto, el sujeto y el predicado no pueden estar al mismo nivel jerárquico.

b) Existen otras funciones (objeto directo, objeto indirecto...) cuya naturaleza es comparable a la función sujeto.

c) Oculta el carácter intercambiable de los actantes. La pasiva supone un reordenamiento del primer actante (del sujeto) y del segundo (del objeto directo).

d) La posición tradicionalista oscurece la teoría de los actantes y de la valencia verbal.

e) Oscurece la teoría de la traslación y de la junción.

A nuestro juicio, estos criterios pueden ser revisados. En primer lugar, no creemos que se deba confundir la omisibilidad ("No iré a la fiesta») con la opcionalidad ("Yo) no iré a la fiesta»). El sujeto siempre está, por tanto, "presente". Además, si no fuera así, ni siquiera se podría considerar el sujeto como un actante más.

Gutiérrez Ordóñez (1997b: 61) arguye que las oraciones impersonales serían un claro contraejemplo a la obligatoriedad del sujeto. Nosotros, en cambio, creemos que la propuesta de Alarcos (1994) fue bastante esclarecedora al diferenciar el sujeto léxico del gramatical. Su defensa puede dar cuenta de estos posibles casos anómalos.

Contra la defensa de la existencia de un sujeto gramatical, Gutiérrez Ordóñez (1997b: 61) encuentra dos críticas contra la defensa del sujeto gramatical. A su parecer, no parece conveniente sostener que los rasgos morfológicos de un verbo cualquiera sean sujeto y a la vez pertenezca al núcleo del predicado. En segundo lugar, Gutiérrez Ordóñez (1997b: 61) afirma que, de este modo, la relación predicativa se establece no sintáctica sino morfológicamente.

A nuestro parecer, cabe recordar que las tesis alarquianas parten de la teoría glosemática. Para Alarcos Llorach (1951 [1969]: 55 y 59), los morfemas verbales son morfemas extensos, esto es, su ámbito de aplicación es heterosintagmática. La concordancia no solo afecta al verbo sino al sujeto. Siendo esto así, es inevitable que el sujeto gramatical esté "en dos bandos". Por otro lado, se ha de tener en consideración que el funcionalismo alarquiano no es la única teoría sintáctica actual que defiende la existencia de un sujeto licenciado por los rasgos morfológicos. $Y$, aunque cada lengua impone sus propias restricciones sintácticas, no se puede obviar el hecho de que, en aquellas lenguas en las que el sujeto es obligatorio, las oraciones impersonales presentan un pronombre expletivo, de significado nulo pero cumple con las exigencias sintácticas de la oración. Finalmente, se ha de tener en consideración que las oraciones impersonales no dejan de ser oraciones marcadas dentro de la gramática del español. Por ello, no es de extrañar que algunos hablantes pronuncien oraciones como «Puede-n haber muchos problemas» o, incluso, una estructura híbrida como "Se cogieron a los ladrones». Estas oraciones son, a nuestro entender, un claro intento de regularizar aquellas oraciones que están marcadas sintácticamente: oraciones sin sujeto. 
Por lo que respecta a establecer la relación predicativa morfológica y no sintácticamente, se ha de indicar, en primer lugar, que hasta el mismo Tesnière asumió que los morfemas verbales pueden ser una expresión del actante sujeto. En concreto, Tesnière (1994 [1954]: 172) llega a afirmar que "la palabra amat es un aglutinado del elemento predicativo amay del elemento sujeto -t. La "separación entre el sujeto y el predicado" no está pues marcada por una separación de palabras". En segundo lugar, se ha de considerar que la expresión de un actante mediante recursos morfológicos no es privativo de la función sujeto. Si se acepta la propuesta de Alarcos Llorach (1994: 198-200), por la que los clíticos o pronombres átonos no son otra cosa que incrementos verbales (esto es, morfemas), no solo la relación sujeto y predicado se establecería mediante recursos morfológicos, sino también el implemento («Lo he visto») o el complemento («Le gustaron mis propuestas»).

El punto (b) afirma que el sujeto es una función equiparable a la del objeto directo o el objeto indirecto. Según nuestro criterio, esta no sería una justificación que desmontaría la estructura bimembre de la oración, sino que es una consecuencia tras la aceptación de que el verbo es el núcleo ordenador de la oración. Por tanto, creemos que la afirmación de (b) no invalida nada.

El punto (c) establece que la pasiva es un claro ejemplo por el que los actantes son intercambiables. A nuestro entender, dependiendo de qué repercusiones sintácticas se entienda que tiene la pasiva, este criterio puede ser acertado o no. Si se entendiera que la pasiva es una simple reordenación de los actantes respecto a un único núcleo, no se explicaría por qué el sujeto de la oración activa («El niño golpeó el balón») pasa a ser un circunstante en la pasiva, esto es, un elemento no exigido («El balón fue golpeado (por el niño)»). Por tanto, no es solo una reordenación actancial como pretende la teoría tesneriana. Mucho más significativo sería su cambio en el caso de las denominadas pasivas reflejas. En este caso, el sujeto de la oración activa desaparece en las oraciones pasivas reflejas («??Se ha golpeado el balón por el niño»). Pero aun aceptando la propuesta tesneriana, no hay que olvidar que, para las tesis funcionalistas, la pasiva no existe en español, sino que las oraciones pasivas serían oraciones atributivas, tesis que parece asumir Gutiérrez Ordóñez (1997: 595-596). Siendo esto así, el denominado complemento agente no puede ser considerado un actante verbal más, sino un mero modificador adjetival. En consecuencia, las tesis del maestro Gutiérrez Ordóñez se encontrarían en las mismas desventajas que la postura tradicional, pues seguiría habiendo una disimetría actancial.

A nuestro juicio, la diátesis supone una auténtica reestructuración oracional y no se observa ningún principio general que impida que esto sea así. Es más, la aceptación de este hecho puede resultar más explicativo que la propuesta tesneriana. Por ejemplo, tal como han puesto de manifiesto diferentes gramáticos y tal como se explica en Tordera Yllescas (2011), la estructura sintáctica de la activa y de la pasiva puede ayudar a explicar el contraste de significado que existe entre la oración «Todos los hombres han amado a una mujer» y «Una mujer ha sido amada por todos los hombres». Una estructura oracional que situara en el mismo nivel sintáctico todos los actantes sería incapaz de poder explicar este contraste de significados. 
El punto (d) afirma que la postura tradicional que defiende la estructura bimembre de la oración puede oscurecer la teoría actancial y la valencia verbal. Nuestra postura es que esto no necesariamente es así. Si se asume que el verbo es el núcleo ordenador de la oración, evidentemente la valencia verbal para un verbo como "dar» solo se puede representar del siguiente modo:

$$
\text { dar }\langle 1,2,3>\quad \text { o bien } \quad \text { dar }<\text { SUJ, OD, Ol }>
$$

Pero por el contrario, si se asume que el sujeto es un argumento externo al verbo, como se ha aceptado desde algunas posturas gramaticales (Haegeman, 1991 [1993]: 83 o Ramos, 1998: 26), se puede aceptar una representación como la siguiente:

$$
<1,<\text { dar, 2, 3>> o bien } \quad<\text { SUJ, }<\text { dar, OD, Ol }>>
$$

Este tipo de representación daría cuenta de cómo los argumentos internos determinan el tipo de sujeto que puede aparecer, mientras que la interpretación tesneriana sería insensible a este hecho. Por ejemplo, mientras que si el verbo «aspirar» exige un OD es posible que el sujeto sea inanimado (v.gr.: «Esta máquina no aspira bien el suelo»), en cambio, cuando exige CRV, solo es posible que el sujeto sea animado (no tiene sentido «La máquina aspira a la victoria»).

Por otro lado, sería debatible hasta qué punto el sujeto es una exigencia léxica (como puede ocurrir con el resto de actantes verbales) o bien es una exigencia sintáctica: toda oración debe tener un sujeto. De hecho, si se acepta este último hecho, se podría defender una estructura argumental como la siguiente:

$<$ dar, 2, 3> o bien <dar, OD, Ol>

dado que sería redundante especificar en el léxico aquello que ya impone la sintaxis.

Finalmente, no consideramos que la estructura bimembre de la oración atente contra la teoría de la junción o de la traslación. Como se verá infra, la estructura bimembre de la oración puede ser tan explicativa como la teoría tesneriana ante oraciones como «Félix limpió y requetelimpió la alfombra» o "Juan bailó y cantó una hermosa canción durante toda la fiesta de cumpleaños».

Respecto a la traslación, esta no se vería sensiblemente alterada. Bien es cierto que existirían traspositores para las categorías sustantivas, adjetivas y adverbiales, pero no traspositores propiamente verbales, sino oracionales. Pero esto no puede ser de otra manera, ya que, como se ha indicado, en el verbo finito recae el núcleo del predicado y los morfemas extensos que expresan el sujeto. Por ello, es posible decir «Juan quiere que vengas», pero no "*Juan quiere que ir.» El traspositor /que $1 /$ solo puede trasponer verbos finitos, esto es, verbos que expresan los morfemas extensos.

Por otro lado, la teoría de Gutiérrez Ordóñez (1997a: 422) tendría que explicitar, por ejemplo, qué elemento traspone el /que 1 . ¿Traspone solo FV ${ }_{1}$ como ocurre en «Juan dijo que tú vendrías"? ¿O traspone solo $\mathrm{FV}_{2}$ como ocurre en "Juan dijo que tú vendrías mañana»? ¿O traspone solo $\mathrm{FV}_{3}$ como ocurre en "Juan dijo que, si podías, tú vendrías»? ¿O traspone solo EL como ocurre en «Juan dijo que, desgraciadamente, vendría Antonio»? 
¿O traspone solo EP como ocurre en «Juan dijo que, por favor, le pasara la sal»? ¿O, sencillamente, el traspositor /que $1 /$ solo puede trasponer el verbo enunciativo como ocurre en «Juan dijo que, francamente, aquella solución no le convencía»? Si fuera esta última la respuesta correcta, nos encontraríamos con que el traspositor /que $1 /$ solo puede trasponer al verbo enunciativo «decir», lo que parece atentar contra la intuición lingüística ${ }^{2}$. En nuestra propuesta, en cambio, el /que ${ }^{1 /}$ solo traspone oraciones.

A nuestro juicio, la oración es una estructura bimembre que consta de un sujeto y un predicado. Y diversas son las razones que pueden apoyar dicha tesis. En concreto, doce pruebas:

a) La prueba de Happ afecta al verbo y a sus argumentos verbales, pero nunca al sujeto. De una oración como "Juan dio un fuerte abrazo a María", se puede decir "Juan lo hizo", pero no «*Lo hizo a María» o «Lo hizo un fuerte abrazo». Esto demostraría que el predicado y el sujeto son dos funciones dispares de la oración (Escandell Vidal, 2004: 142).

b) Se puede focalizar mediante una perífrasis de relativo (una ecuacional) el verbo y sus argumentos (esto es, el predicado), pero no el sujeto y el verbo. Por ello, se puede decir «Lo que hizo Juan fue abrazar a María» pero no «*Lo que hizo a María fue abrazar Juan» (Escandell Vidal, 2004: 142).

c) Cuando un elemento focalizador como «solo» afecta al predicado, puede afectar tanto al verbo como a sus argumentos. Así pues, ante una oración como "Juan solo abrazó a María», se puede interpretar que el adverbio focalizador afecta al verbo (v.gr.: "Juan solo abrazó a María; no la besó») o bien a sus argumentos (v.gr.: "Juan solo abrazó a María y a nadie más»). En cambio, cuando el adverbio incide sobre el sujeto no afecta al predicado (v.gr.: en la oración «Solo Juan abrazó a María» se interpreta de manera no marcada que «Nadie más la abrazó» pero no se puede continuar «??Solo Juan abrazó a María pero no la besó») (Escandell Vidal, 2004: 142-143). Bien es cierto que, en oraciones tales como «A María solo la abrazó Juan», puede interpretarse tanto «solo la abrazó, no la besó», como «solo Juan, nadie más». Pero cabe observar que, en este caso, se trata de una oración marcada respecto al orden de las palabras, ya que no es SVO${ }^{3}$. Por ello, el complemento adquiere características que le son propias al sujeto. En primer lugar, el orden del complemento es antepuesto al verbo. Y, en segundo lugar, necesariamente, se ha de dar la concordancia objetiva: el clítico «la» se hace obligatorio. Por tanto, es evidente que el complemento verbal se "subjetiviza".

d) Según Escandell Vidal (2004: 143), se puede «coordinar fácilmente dos predicados con el mismo sujeto», como ocurriría en la oración «Juan [admira a María] y [ama a Claudia]»;

\footnotetext{
2 Por otro lado, sería cuestionable la catálisis de un verbo enunciativo en el ejemplo propuesto: «??Juan dijo que (yo digo que), francamente, aquella solución no le convencía». Esto demostraría que este presunto verbo enunciativo, que transmite las coordenadas enunciativas (el aquí y ahora enunciativo), no puede estar nunca traspuesto a ningún elemento. Solo puede encabezar la oración (i.e., "(Yo digo que) Juan dijo que, francamente, aquella solución no le convencía») y no puede depender de ningún otro. Por tanto, no se podría trasponer el presunto verbo enunciativo.

${ }^{3}$ Como se indica en Padilla (2003), el orden oracional del español no está determinado solo por factores sintácticos, sino que los factores pragmáticos desempeñan un papel fundamental. En cualquier caso, nuestra tesis es que el orden sintáctico no marcado del sujeto tiende ser el antepuesto al verbo, salvo que operen otros factores sintácticos como la inacusatividad.
} 
sin embargo, la coordinación del sujeto y un verbo sin que dicha relación afecte al resto de complementos verbales es agramatical («"Juan admira] y [Pedro ama] a María») o, cuando menos, se trata de una estructura muy marcada para el español («? Juan admira, y Pedro ama, a María»).

e) En la composición morfológica, es frecuente formar nuevas palabras a partir de la estructura sintáctica verbo+complementos («sacacorchos", "parabrisas»...), pero no a partir del sujeto+verbo. Por ejemplo, no existe un compuesto como «limpiacrema» o "Cremalimpia» (Escandell Vidal, 2004: 143). Lo mismo se podría decir sobre las unidades fraseológicas. Existen unidades fraseológicas que presentan el predicado fosilizado (v.gr.: ( «Juan/el niño/el idiota... estiró la pata», "Juan/el niño/el idiota... nos dio gato por liebre», etc.), pero no se conocen unidades fraseológicas en las que solo se fosilicen el sujeto y el verbo sin sus complementos.

f) Los cuantificadores flotantes solo son posibles cuando el sintagma nominal funciona como sujeto (Bel, 2002: 1089): «(Todos) los alumnos asumieron (todos) su parte de responsabilidad». Cuando el sintagma nominal desempeña otra función, los cuantificadores pierden su movilidad: «Los alumnos hablaron con todos sus profesores».

g) Las formas verbales no personales o verboides presentan los mismos complementos verbales que las formas finitas (salvo el objeto directo en el caso del participio, debido a su valor pasivo). En cambio, la aparición de un sujeto léxico explícito está supeditada a fuertes restricciones sintácticas (Bel, 2002: 1088), pues en determinados contextos puede aparecer («Al llegar la primavera, los soldados marcharon», «Aun siendo Juan tan listo, suspendió el examen», «Muerto el perro, se acabó la rabia»), pero en otros, su presencia es rechazada (v.gr.: "Antonio deseaba comprarse un bajo cerca de su casa", "Vi a Miguelito saltando sobre el charco», «Todavía conserva la ropa donada por Andrés a la parroquia de su pueblo»).

h) El sujeto presenta el caso no marcado en las lenguas nominativo-acusativas (Bel, 2002: 1087-1088). El castellano, al no ser una lengua con declinaciones, la marca de caso solo subsiste en los pronombres (v.gr.: "yo» vs «mí»). Sin embargo, a pesar de que no existan rasgos morfológicos, sí perviven sus efectos sintácticos. Por ejemplo, Moreno Cabrera (1994) señala que, en una oración coordinada del tipo "Juan golpeó a Pedro y $\varnothing$ se marchó», la lectura no marcada sería aquella en la que Juan es el que golpea y se marcha frente a la lectura marcada en la que Juan golpea a Pedro y este último se marcha. En cambio, ocurre lo contrario en las lenguas ergativas, en las que el sujeto de las oraciones transitivas es el elemento marcado (Moreno Cabrera, 1994: 59-60).

i) Ante la elisión del verbo, es la presencia del sujeto lo que nos permite seguir hablando de una oración y no la presencia de otros complementos. En la oración "Juan se tomó unas cervezas y Pedro, dos copas de vino", se defendería una catálisis del verbo «tomó» en la segunda oración, «Pedro, dos copas de vino», solo por la presencia del sujeto «Pedro». En cambio, no serían pocos los gramáticos que negarían la necesidad de realizar dicha catálisis en oraciones como «Juan se tomó unas cervezas y (también) dos copas de vino». Lo mismo ocurriría con una oración del tipo «Juan se tomó unas cervezas y Pedro 
también». Igualmente, se propondría en este caso la existencia de dos oraciones. Consecuentemente, como no puede haber sujeto sin predicado, la presencia de aquel legitima hablar de una oración.

j) Solo si se asume que la oración es la unión de un sujeto y un predicado, se pueden abordar con éxito oraciones como «Los camareros estuvieron yendo y viniendo». A nuestro juicio, esta oración es una oración simple, independientemente del número de verbos implicados. La razón estribaría en el hecho de que solo se puede defender la existencia de un único sujeto; la catálisis de un segundo sujeto sería, a nuestro juicio, un recurso ad hoc, con el fin de mantener la creencia de que siempre que hay verbo, hay oración. Además, la posible catálisis generaría una oración con un sentido diferente al de la oración original: «??Los camareros estuvieron yendo y los camareros estuvieron viniendo».

k) En Bel (2002: 1087), se indica que un rasgo discriminativo de la función sujeto frente al resto de complementos verbales es el de la concordancia. A nuestro juicio, esta es una afirmación que, aunque cierta, necesita ser matizada. Asumir que solo el sujeto es aquel que concuerda con el verbo nos llevaría a negar la existencia de la concordancia objetiva en castellano (v.gr.: «A Juan lo vi a ayer» O «A mí no me gustan las lentejas»). Además, interlingüísticamente, no es cierto tampoco que solo el sujeto concuerde con el verbo. Por ejemplo, en vasco, el sujeto, objeto directo e indirecto pueden concordar con el verbo. Lo que sí han puesto de manifiesto diferentes autores desde teorías sintácticas dispares (Gazdar, Klein, Pullum y Sag 1985: 198; Pollard y Sag 1987: 117-121; Bresnan 2001: 212213) es que el sujeto es una función jerárquicamente superior al resto de funciones, lo que tiene sus repercusiones sintácticas. Por ejemplo, si en una lengua dada existe una función que concuerda con el verbo, esta función debe ser el sujeto. Por tanto, no se puede dar la concordancia verbal con un objeto directo, indirecto o complemento de régimen sin que no se dé la concordancia con el sujeto. Esto es lo que ocurre en castellano. La concordancia verbal con el sujeto está totalmente gramaticalizada; en el resto de funciones (objeto directo e indirecto), estaría en vías de gramaticalización (dado el carácter seudoafijal de los clíticos y que la concordancia con el objeto directo está ligada a la posición, al menos, en el español peninsular).

I) Existen diversos fenómenos sintácticos como la elisión, la reflexividad/reciprocidad, la pasividad, la impersonalidad, la inacusatividad... que afectan especialmente al sujeto. Sin embargo, no conocemos fenómenos sintácticos de diátesis que generen o hagan desaparecer objetos indirectos o complementos de régimen verbal. Sí se puede dar el caso de la omisión de objetos directos (v.gr.: "Juan come manzanas» y "Juan come»), pero se ha de indicar que dicho fenómeno no es generalizable y está subordinado, en cualquier caso, a otros hechos sintáctico-semánticos como el Aktionsart o aspecto léxico. Cuando el verbo es delimitado o télico, la omisión suele dar resultados agramaticales; por ejemplo, se puede decir "Juan comió (manzanas)», con elisión del objeto directo, pero no "Juan se comió *(las manzanas)». Lo mismo cabe decir con verbos de significado similar: no es posible omitir el objeto directo en oraciones como «Juan devoró *(el plato)». En cambio, la posibilidad de omitir el sujeto no parece obedecer en ningún caso al aspecto léxico o cualquier otro fenómeno sintáctico. Por otro lado, también hay que hacer notar que oraciones del tipo «Habían muchas casas» (mayoritarias del Levante y sur de España así 
como en algunas regiones hispanoamericanas) u otras como "se cogieron a los ladrones» muestran la tendencia de la lengua a regularizar oraciones marcadas dentro del sistema: oraciones con un único actante/argumento distinto al sujeto. Lo no marcado es que si solo existe un actante verbal este corresponda con el sujeto. Estas oraciones ejemplificadas muestran, a nuestro juicio, un caso de regularización de lo excepcional.

Así pues, aunque sea cierto que la estructura bimembre de la oración tuviera en sus orígenes una motivación extralingüística (en concreto, logicista), tal como apuntó Gutiérrez Ordóñez (1997b), a tenor de los rasgos indicados, no se puede negar que existen suficientes causas lingüísticas para defender el carácter bimembre de la oración.

\section{NUESTRA PROPUESTA}

Al aceptar que la oración es una estructura bimembre, en consecuencia, hemos de desechar la jerarquía oracional propuesta por Gutiérrez Ordóñez (1997a). A nuestro juicio, una adecuada jerarquía oracional debe dar cuenta de la diferencia entre los elementos exigidos y los no exigidos; entre el sujeto y el resto de complementos y entre las funciones oracionales y las funciones extraoracionales.

Si se analiza una oración como «Juan piensa en la cárcel», se puede observar que esta puede tener dos interpretaciones según se asigne «en la cárcel» la función de complemento de régimen («En aquello en lo que piensa es en la cárcel») o bien un circunstancial («En donde piensa es en la cárcel»). Dado que el complemento de régimen es un argumento del verbo, debe estar próximo a este; en cambio, los denominados circunstanciales deben modificar a todo el predicado. La jerarquía oracional propuesta por cualquier teoría sintáctica actual debe dar cuenta de este hecho:

(1)

a.

$$
\begin{array}{ll}
\text { Juan piensa en la cárcel. } & \frac{\frac{\mathrm{N}}{\text { SUPLEM }}}{\text { SUJ }} \frac{}{\text { PREDICADO }}
\end{array}
$$

b.

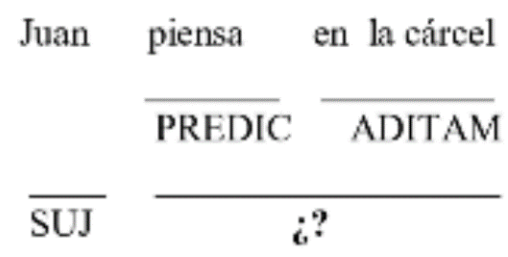

Las representaciones ofrecidas darían cuenta de la distinta jerarquía oracional de los complementos exigidos frente a los no exigidos. No obstante, esta propuesta parece generar un nuevo problema: ¿cuál es la función que domina al predicado y al complemento circunstancial?

En la tradición gramatical hispánica, ha sido habitual crear una nueva etiqueta para funciones o categorías que se presentan como jerárquicamente superiores a otras. Por ejemplo, Rojo (1978) hablaba de cláusulas y oraciones; Hernández Alonso (1996 [1984]), de forma similar, diferenciaba entre nexus y oraciones. Incluso, Gutiérrez Ordóñez (1997b), que reduce las cláusulas, nexos, proposiciones y oraciones a la frase verbal (pues 
clásulas/nexus/proposiciones = oraciones y oración = frase verbal), se ve obligado a hablar de enunciado lingüístico y de enunciado pragmático (Gutiérrez Ordóñez, 1997a) como categorías jerárquicamente superiores a la frase verbal.

Sin embargo, existe una segunda opción en sintaxis: dados dos funciones, A y B, que forman una única función, esta recibirá el nombre de la función nuclear. Según este criterio, la función resultante de un predicado y un circunstancial volvería a ser un predicado. Esta parece ser también la postura de Gutiérrez Ordóñez (1997a) al proponer la FV', la FV² y la $\mathrm{FV}^{3}$.

Aceptando la propuesta de que una función se puede iterar $n$ veces, se puede seguir manteniendo que la oración es la unión de un sujeto y un predicado en los casos en los que existen aditamentos. Solo se ha de iterar la función predicado en aquellos casos en los que aparece al menos un aditamento. El aditamento modificaría al todo el predicado y la función resultante volvería a ser un predicado. $\mathrm{Y}$, al ser la oración una estructura bimembre (y, por tanto, el núcleo no es solo el predicado o el verbo), el predicado cedería paso a una nueva función: la función oración.

Por otro lado, el hecho de que una función se reitere de forma jerárquica no es una novedad nuestra, sino que ya está de forma implícita en el funcionalismo alarquiano y puede dar cuenta de construcciones que pueden resultar problemáticas para otros marcos teóricos. Alarcos (1980 [1970]: 273) afirmó que las denominadas conjunciones copulativas (y, en general, cualquier conjunción coordinativa de la gramática tradicional) une elementos equifuncionales. Siendo esto así, el análisis propuesto para oraciones como «María y su hijo se han ido", "Félix limpió y requetelimpió la alfombra» o "Antonio bailó y cantó una hermosa canción durante toda la fiesta de cumpleaños» podría ser el siguiente:

(2)

a.

María y su hijo se han ido

$\overline{\mathrm{SUJ}} \overline{\mathrm{Nx}} \overline{\mathrm{SUJ}}$

SUJETO $\overline{\text { PREDIC }}$

b.

$$
\begin{aligned}
\text { Félix limpió } \mathrm{y} \text { requetelimpió la alfombra } \\
\frac{\frac{\mathrm{Núc}}{\mathrm{Nx}} \frac{\mathrm{Núc}}{\text { Núcleo }}}{\text { SUJ }} \frac{}{\text { IMPLEM }} \\
\frac{\text { PREDICADO }}{}
\end{aligned}
$$


c.

Antonio bailó $\mathrm{y}$ cantó una hermosa canción durante toda la fiesta de cumpleaños
$\frac{\overline{\text { Núcl }} \frac{}{\text { PRE }} \overline{\mathrm{Nx}} \frac{\text { Núcl }}{\text { PREDICADO }}}{\text { PREDICADO }}$
$\frac{\text { PREDICADO }}{\text { SUJ }} \frac{\text { ADITAMENTO }}{\text { PRLM }}$

En el análisis de (2a), siguiendo las directrices alarquianas, la conjunción /y/ ha unido dos elementos equifuncionales (dos sujetos) y el resultado no puede ser otro salvo la función sujeto. En (2b), dado que tanto el verbo «limpiar» como el verbo «requetelimpiar» exigen un implemento, es lógico proponer la coordinación de dos núcleos verbales (como se pueden unir dos núcleos sustantivos, "Cogió revistas y libros viejos»). Además, semánticamente, la acción de «limpiar y requetelimpiar» se percibe de manera unitaria y esto es lo que trata de recoger el análisis. Finalmente, en (2c), dado que en la lectura no marcada se interpreta que alguien realiza dos acciones durante una fiesta de cumpleaños, el aditamento, «durante toda la fiesta de cumpleaños» debe afectar al predicado compuesto «bailó y cantó una hermosa canción». En cualquiera de los tres análisis, como se ve, ha sido imprescindible que se reitere una función (sea el sujeto, el núcleo del predicado o el predicado). Por tanto, parece acertado el análisis propuesto.

Por otro lado, quedaría la cuestión de en qué nivel sintáctico se situarían los circunstantes, los marcadores de modalidad lingüística y pragmática así como los aditamentos y circunstantes del verbo enunciativo. Dado que parecen elementos extraoracionales, una opción sería establecer diferentes niveles oracionales, $\mathrm{O}^{1}, \mathrm{O}^{2}, \mathrm{O}^{3} \ldots$, (al igual que Gutiérrez Ordóñez (1997a) propone $\mathrm{FV}^{1}, \mathrm{FV}^{2}, \mathrm{FV}^{3}$ ), sobre el que incidirían las distintas funciones extraoracionales de manera jerárquica.

(3)

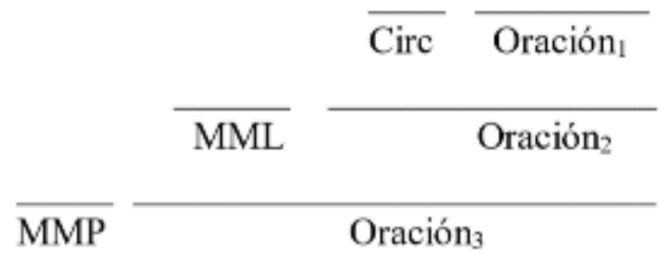

\begin{tabular}{lll} 
& $\overline{\text { ADIT Ven }}_{\text {Cir Ven }}$ & Oración $_{4}$ \\
& & Oración $_{5}$ \\
\hline
\end{tabular}

\section{Oración $_{6}$}


Sin embargo, la cuestión no parece resolverse de manera fácil. La mayoría de gramáticos no proponen más que un tipo de función extraoracional. De hecho, no parecen factibles oraciones en las que aparezcan juntos algunos de estos elementos extraoracionales: «??Francamente/con franqueza (AditVen), desgraciadamente (MML o Atr. Orac), todo ha acabado", «? En el banco (Circunstante), ¿acaso (MMP) no tiene dinero usted?», (parece preferible «¿Acaso no tiene usted dinero en el banco?») y cualquier MML es incompatible con un MMP, pues los primeros nunca pueden aparecen con una oración que no sea enunciativa afirmativa, («*Posiblemente, (MML), ¿acaso (MMP) va a venir Juan?») ${ }^{4}$. Menos posible parece el que una oración contenga un ejemplo de cada función extraoracional, (aunque sí existen oraciones con sujetos, implementos, suplementos y aditamentos...). No parece posible la presencia múltiple de las diferentes funciones extraoracionales mencionadas (aunque sí se puedan reiterar una misma función extraoracional). Por otro lado, los criterios de determinación de algunas funciones son algo cuestionables. Por ejemplo, «probablemente» sería un MML (o antiguo atributo oracional) porque admite la paráfrasis «es probable que O». Sin embargo, otros elementos como «quizás» parecen tener el mismo comportamiento que «probablemente» (no solo significan prácticamente lo mismo sino que ambos han de aparecer con el verbo en subjuntivo), pero no admiten dicha paráfrasis. Además, teniendo en cuenta que no faltan gramáticos que considerarían que estos elementos introducen una oración dubitativa, quizás sería más acertado considerar tanto «quizás» como «probablemente» como MMP. Finalmente, la existencia de un verbo enunciativo implícito como «decir», (con una influencia clara de la teoría defendida por Ross), no está exenta de problemas (Escandell Vidal, 1996: 69-70). Es difícil la defensa de un verbo enunciativo para algunas oraciones (v.gr.: «Eres un imbécil» >> «??Te digo que eres un imbécil») así como para los actos de habla indirectos. (v.gr.: "¿Me puedes pasar la sal?» >> «¿Te digo que si me puedes pasar la sal?»). La catálisis de un verbo enunciativo haría que el enunciado perdiera la fuerza ilocutiva en un contexto de habla real o resultase anómalo desde un punto de vista pragmático.

A nuestro juicio, al igual que no es usual que un mismo verbo tenga un implemento, un suplemento y un complemento o que un número elevado de aditamentos afecten a un mismo predicado, quizás estos problemas se resolverían si se aceptara que todas estas funciones pueden modificar a la función oración, pero también parecen existir ciertas restricciones numéricas (a partir de dos elementos, parece saturarse la oración) y de incompatibilidades funcionales (ciertas funciones no pueden coaparecer). Por ello, nuestra propuesta para una jerarquía oracional sería la siguiente:

(4)

\footnotetext{
${ }^{4}$ Es cierto que estos elementos no se pueden coordinar entre sí, pero también lo es que tampoco se pueden coordinar entre sí un implemento y un suplemento, o un sujeto y un aditamento. La imposibilidad de coordinar un MML y un MMP solo indica que podemos estar ante funciones distintas, pero no ante funciones que pertenezcan a una jerarquía sintáctica distinta. De hecho, el que el MML y el MMP sean incompatibles entre sí, podría sugerir que estamos ante funciones que pertenecen en el mismo nivel jerárquico. Si bien se puede decir "Juan piensa el problema» y "Juan piensa en la solución", lo que no se puede decir es «"Juan piensa el problema y en la solución». Esto solo demuestra que el implemento y el suplemento son funciones distintas, pero no que estas funciones pertenezcan a un nivel sintáctico diferente.
} 


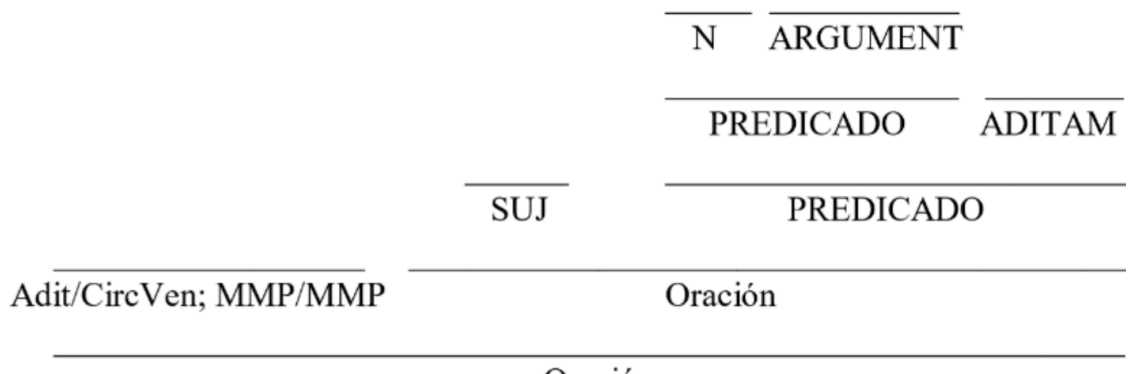

Oración

Respecto a los conectores textuales de Gutiérrez Ordóñez (1997a), como se puede observar, estos elementos no han sido recogidos en nuestra jerarquía oracional. La explicación es sencilla. Tal como se explica en Briz et alii (2003), los conectores textuales son elementos que no solo inciden solo sobre la oración, sino que pueden incidir más allá del ámbito oracional. Por tanto, estamos ante unidades ante las cuales la sintaxis oracional se revela ineficaz y es necesario recurrir a teorías supraoracionales.

Finalmente, es posible que haya quien considere que lo que aquí se presenta es una reelaboración funcionalista de los principios generativistas (Chomsky, 1981 y 1995). En parte, hay ciertas semejanzas, a saber, la reiteración de una función ante funciones no argumentales.

(5)

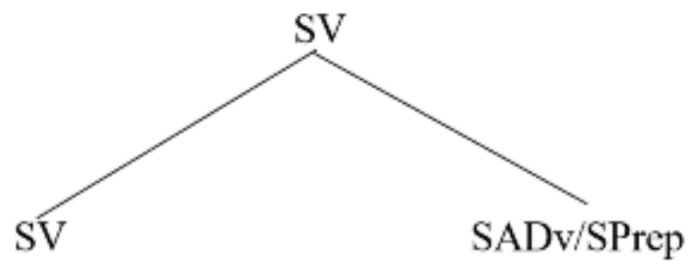

Pero también hay notables diferencias. Nosotros no defendemos el binarismo de la X-barra. En unas oraciones como "Juan dio a María un regalo" y "Juan dio un regalo María», existen dos funciones argumentales (implemento y complemento) que modifican al verbo de idéntica forma y situados en la misma jerarquía. A nuestro juicio, no hay necesidad de proyectar en diferentes niveles jerárquicos el implemento, complemento y suplemento so pretexto de mantener el binarismo sintáctico. Lo mismo ocurriría con oraciones del tipo «Juan durmió diez horas en el hotel» y «Juan durmió en el hotel diez horas». Nosotros solo apreciamos dos aditamentos que afectan al predicado. El generativismo chomskiano, en contraste, se vería obligado a proyectar tantos SV como complementos no exigidos hubiera. Si respetamos la navaja de Ockham (en igualdad de condiciones, la teoría más simple es la mejor), nuestra propuesta parece más adecuada, pues es más sencilla y no parece que sea más explicativo proyectar SV ad infinitum. Por otro lado, el binarismo generativista sería incapaz de analizar de forma satisfactoria oraciones como «Félix limpió y relimpió la alfombra», pues cada $V$ ha de proyectar un SV y cada elemento léxico ha de 
satisfacer sus exigencias argumentales (como la de regir un SN si es un verbo transitivo). Asimismo, el generativismo tendría problemas a la hora de analizar otras oraciones como «Los niños y las niñas más inteligentes se enfrentarán a ese problema». En este caso, el SAdj «más inteligentes» parece adjuntarse a los dos SN, pues afecta a ambos elementos (esto es, a «niños» y a «niñas»). Si se parte de un Sintagma Determinante como parece proponer el generativismo, no se puede dar cuenta de este tipo de oraciones.

Por tanto, nuestra propuesta sí presenta ciertas semejanzas con la propuesta generativista, pero rechaza la rigidez impuesta por la teoría de la X-barra así como el análisis categorial en detrimento del funcional. Es decir, rechaza la esencia misma de las gramáticas generativas.

En conclusión, hemos de defender la estructura bimembre de la oración a tenor de las doce pruebas aportadas. Esto nos ha obligado a realizar un replanteamiento de la propuesta del maestro Salvador Gutiérrez. En nuestra propuesta, los argumentos y el núcleo del predicado forman un predicado que puede, a su vez, ser modificado por los distintos complementos verbales no obligatorios. La función resultante es el predicado. $Y$ el sujeto y el predicado forman la oración. Dicha función, puede ser modificada por distintas funciones extraoracionales. Aunque nuestra tesis no es definitiva (pues las funciones extraoracionales siguen siendo una cuestión no cerrada todavía), nuestra creencia es que, aunque las distintas funciones extraoracionales defendidas por Salvador Gutiérrez pueden presentan unas características sintácticas definitorias dadas, lo cierto es que también presentan unas propiedades propias los distintos argumentos verbales (implemento, suplemento o complemento) y no por ello se propone un nivel sintáctico distinto por cada una de estas funciones (como el propio Salvador Gutiérrez parece aceptar). Así pues, no creemos que sea conveniente proponer un nivel sintáctico distinto para cada función extraoracional. No deslegitimamos la defensa de estas funciones, pero sí se cuestionan tantos niveles sintácticos, sobre todo, si se tiene en cuenta que existen funciones, como la MML y la MMP, que no pueden coaparecer en una oración.

\section{BIBLIOGRAFÍA}

AlARCOS LloRACH, Emilio (1980 [1970]): Estudios de Gramática Funcional del Español, Madrid, Gredos, $3^{a}$ edición.

AlARCOS LLORACH, Emilio (1951 [1969]): Gramática estructural, Madrid, Gredos, $2^{a}$ edición.

AlarCos LloRACH, Emilio (1994): Gramática de la Lengua Española, Madrid, Espasa-Calpe.

BEL, Aurora (2002): «Les funcions sintàctiques», en Lloret, Maria Rosa, Joan MAscaró y Manuel Pérez Saldanya, coord., Gramàtica del Català Contemporani, Barcelona, Empúries, 10751147.

BRESNAN, Joan (2001): Lexical-Functional Syntax, Oxford, Basil Blackwell.

BRIZ, Antonio et alii (2003): "Un sistema de unidades para el estudio del lenguaje coloquial», Oralia, 6, 7-61.
CHOMSKY, Noam (1981): Lectures on Government and Binding, Dordrecht, Foris.

CHOMSKY, Noam (1995 [1998]): El programa minimista. Els escrits de Chomsky, Barcelona, Ariel.

ESCANDELL VIDAL, Mª. Victoria (1996): Introducción a la pragmática, Barcelona, Ariel.

ESCANDELL VIDAL, Mª. Victoria (2004): Fundamentos de Semántica composicional, Barcelona, Ariel.

Gazdar, Gerald, Ewan KLeIn, Geoffrey Pullum e Ivan A. SAG (1985): Generalised Phrase Structure Grammar, Oxford, Blackwell/Cambridge University press.

GUTIÉRREZ ORDÓÑEZ, Salvador (1997a): «La determinación de los niveles oracionales", $L a$ oración y sus funciones, Madrid, Arco/Libros, 368-426. 
GUTIÉRREZ ORDÓÑEZ, Salvador (1997b): «¿Es necesario el concepto de "oración"?", La oración y sus funciones, Madrid, Arco/Libros, 40-65.

GUTIÉRREZ ORDÓÑEZ, Salvador (1997c): "Comentario sintáctico", La oración y sus funciones, Madrid, Arco/Libros, 577-598.

HAEGEMAN, Liliane (1991 [1993]): Teoria de la recció i el lligam, Barcelona, Enciclopèdia Catalana.

HeRnÁndeZ Alonso, César (1996 [1984]): Gramática funcional del español, Madrid, Gredos.

LÓPEZ GARCÍA-Molins, Ángel (1996): Gramática del español. II La oración simple, Madrid, Arco/Libros.

Martínez, José Antonio (1994a): Propuesta de gramática funcional, Madrid, Istmo.

MARTínEZ, José Antonio (1994b): Funciones, categorías y transposición, Madrid, Istmo.

MARTíneZ, José Antonio (1994c): Cuestiones marginadas de gramática española, Madrid, Istmo.
Moreno CABRERA, Juan Carlos (1994): Fundamentos de Sintaxis General, Madrid, Síntesis.

RAmos AlFAJARIN, Joan Rafael (1998): Introducció a la sintaxi, Valencia, Tàndem.

RoJo, Guillermo (1978): Cláusulas y oraciones, Anejo de Verba, 14, Santiago de Compostela, Servicio de Publicaciones de la Universidad de Santiago.

PADILLA, Xose A. (2003): El orden de palabras en el español coloquial, Tesis Doctoral, València, Universitat de València.

PollaRD, Carl e Ivan A. SAG, (1994): Head-Driven Phrase Structure Grammar, Chicago, Chicago University press.

TESNIĖRE, Lucien (1994 [1954]): Elementos de sintaxis estructural, Madrid, Gredos.

TORDERA YLLESCAS, Juan Carlos (2011): «El ámbito de los cuantificadores I. Lo que la gramática sí codifica", Miscelánea lingüística. Cuestiones de sintaxis, semántica y enseñanza del español, Paiporta, Ediciones La sirena, 51-60. 